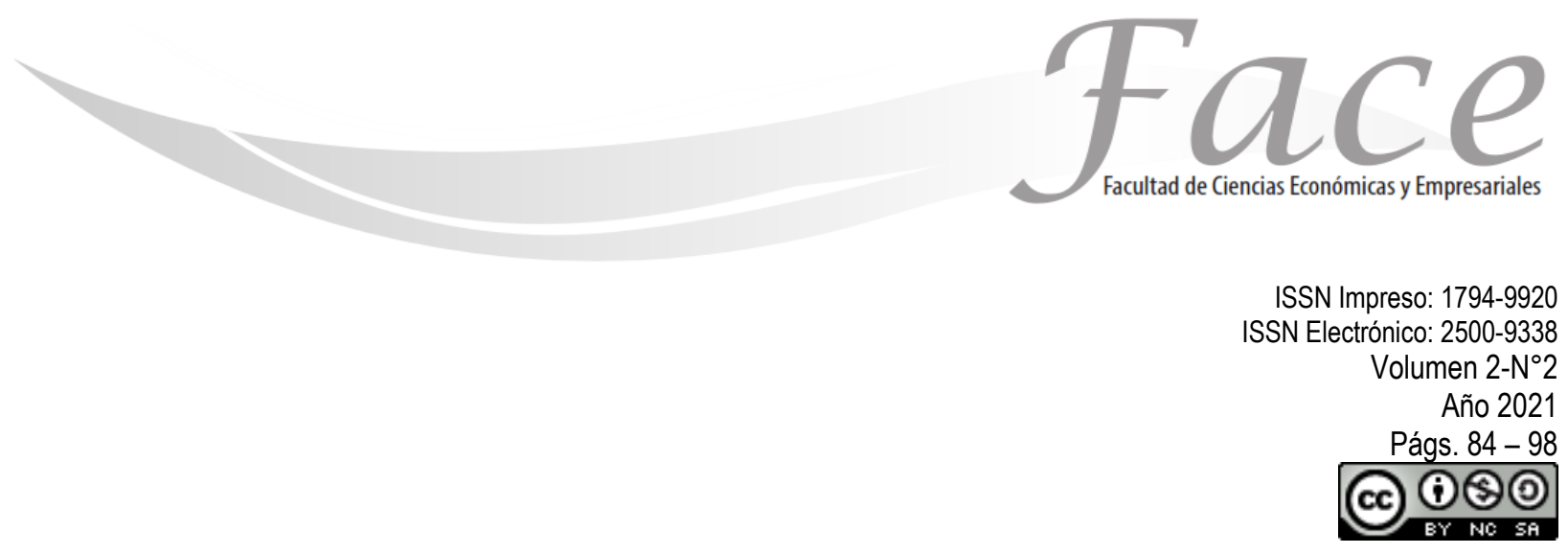

\title{
ANÁLISIS DE ESTRATEGIA EN REDES SOCIALES PARA EL SECTOR DE LOS RESTAURANTES COLOMBIANOS EN TIEMPOS DE CRISIS POR LA PANDEMIA COVID-19
}

\author{
Yenny Katherine Parra Acosta \\ Enlace ORCID: https://orcid.org/0000-0001-6004-2796 \\ Carlos Alberto Almanza \\ Enlace ORCID: https://orcid.org/0000-0002-4561-4941 \\ Diego Felipe Astudillo Valverde \\ Enlace ORCID: https://orcid.org/0000-0003-3064-9380
}

Fecha de Recepción: Febrero 19

Fecha de Aprobación: Mayo 21

\section{Resumen:}

El mercadeo en redes sociales es una de las tendencias más importantes en la actualidad, ya que las empresas, incluidos los restaurantes han tenido que migrar a los entornos digitales para sobrevivir a la crisis causada por la pandemia Covid-19. El objetivo de este documento es analizar la estrategia de los restaurantes colombianos en las dos redes sociales con mayor Retorno sobre la inversión en Latinoamérica: Facebook e Instagram (Hubspot, 2021), y realizar un análisis de correlación entre las variables seleccionadas permitiendo así definir, mejorar e informar acerca de las mejores prácticas en el sector. Para alcanzar este objetivo se recolectó por medio de netnografía la información de una muestra de 146 restaurantes colombianos, y se analizaron de forma general, para posteriormente hacer una división entre franquiciado o no, esto último con la intención de conocer si hay fortalezas en la estrategia en redes sociales que las diferencian de los restaurantes que no lo son, y así determinar si cuentan con mejores prácticas que puedan ser adoptadas por el sector.

Palabras clave: Marketing relacional digital, Redes sociales, Restaurantes.

\footnotetext{
* Profesora de la Facultad de Ciencias Económicas, Universidad Militar Nueva Granada, Sede Campus, Colombia. Doctora en Gestión, Magíster en Administración de Empresas, especialista en Mercadeo y Ventas. Profesional en Administración de empresas. Investigadora Asociada de Colciencias. Correo electrónico: yenny.parra@unimilitar.edu.co

** Profesor de la Facultad de Ciencias Económicas, Universidad Militar Nueva Granada, Sede Campus, Colombia. Magíster en Administración, especialista en Pedagogía y Docencia Universitaria. Profesional en Administración de empresas e Ingeniería Industrial. Investigador categorizado como Estudiante de Doctorado por Colciencias. Correo electrónico: carlos.almanza@unimilitar.edu.co

*** Profesor de la Facultad de Ciencias Económicas y Administrativas, Universidad Uniagustiniana, Bogotá, Colombia. Magíster en Educación, especialista en Mercadeo de Servicios y Neuromarketing. Profesional en Administración de empresas. Correo electrónico: diego.astudillo@uniagustiniana.edu.co
} 


\title{
STRATEGY ANALYSIS IN SOCIAL MEDIA FOR THE COLOMBIAN RESTAURANT SECTOR IN TIMES OF CRISIS DUE TO THE COVID-19 PANDEMIC
}

\begin{abstract}
:
Social media marketing is one of the most important trends today, as companies, including restaurants, have had to migrate to digital environments to survive the crisis caused by the Covid-19 pandemic. The objective of this document is to analyze the strategy of Colombian restaurants in the two social networks with the highest Return on investment in Latin America: Facebook and Instagram (Hubspot, 2021), and to perform a correlation analysis between the selected variables, thus allowing defining, improve and inform about best practices in the sector. To achieve this objective, the information from a sample of 146 Colombian restaurants was collected through netnography, and they were analyzed in a general way, to later make a division between franchisees or not, the latter with the intention of knowing if there are strengths in the strategy in social networks that differentiate them from restaurants that are not, and thus determine if they have best practices that can be adopted by the sector.
\end{abstract}

Keywords: Digital relational marketing, Social networks, Restaurants.

\section{ANÁLISE DE ESTRATÉGIA EM REDES SOCIAIS PARA O SETOR DE RESTAURANTES LOS COLOMBIANOS EM TIEMPOS DE CRISIS BY PANDEMIA COVID-19}

Resumo:

O marketing de mídia social é uma das tendências mais importantes hoje, já que as empresas, incluindo restaurantes, tiveram que migrar para ambientes digitais para sobreviver à crise causada pela pandemia Covid-19. $O$ objetivo deste documento é analisar a estratégia dos restaurantes colombianos nas duas redes sociais com maior Retorno do investimento na América Latina: Facebook e Instagram (Hubspot, 2021), e realizar uma análise de correlação entre as variáveis selecionadas, permitindo assim definir, aprimorar e informar sobre as melhores práticas do setor. Para atingir esse objetivo, foram coletadas informações de uma amostra de 146 restaurantes colombianos por meio da netnografia, as quais foram analisadas de forma geral, para posteriormente fazer a divisão entre franqueados ou não, este último com o intuito de saber se há pontos fortes em a estratégia nas redes sociais que os diferenciam dos restaurantes que não o são, e assim determinam se possuem as melhores práticas que podem ser adotadas pelo setor.

Palavras-chave: relacional, Redes sociais, Marketing digital Restaurantes. 


\section{INTRODUCCIÓN:}

El marketing digital se convirtió en una de las áreas imperativas para las organizaciones empresariales de todo el mundo (Patro, 2021). El fenómeno de las redes sociales ha transformado la forma en que nos comunicamos e interactuamos con nuestro entorno. Para 2020, el número de usuarios activos de redes sociales alcanzó los 3,8 mil millones, con una penetración media mundial del 49\% (Matosas-López, 2021). En ese contexto, las redes sociales han pasado de ser medios de información a medios de comunicación por excelencia, posicionándose como una poderosa herramienta de mercadeo, sin embargo, para utilizarlos de forma eficaz es necesario conocer sus usos, funciones y buenas prácticas de tal forma que se optimice la inversión. En la presente investigación, se realizó un análisis netnográfico de las estrategias implementadas por los restaurantes colombianos en las redes sociales, principalmente los que tienen presencia en Facebook e Instagram, para de esta manera tratar de determinar las mejores prácticas en el sector.

En el entorno que se vive en medio de la pandemia, las empresas tuvieron que encontrar alternativas de acción para intentar sobrevivir. En ese sentido, los restaurantes tuvieron que buscar estrategias que les permitiera sobrellevar la difícil situación obligados por el cierre obligatorio de los establecimientos lo que produjo una fuerte caída de los ingresos y en los casos más extremos al cierre de los restaurantes. En este sentido, por motivo de la pandemia cerraron más de 30.000 restaurantes, representado en su mayoría por microempresas y establecimientos informales (Semana, 2021).

En esta revisión netnográfica se tienen en cuenta variables como los seguidores con los que cuenta la red social del restaurante, la información que brinda y la satisfacción que puede llegar a tener el cliente cuando revisa aquellos portales de internet, con el fin de destacar los aspectos positivos y los posibles aspectos de mejora que se deben realizar para lograr impactar al público de una forma más efectiva.

Se utilizó la netnografía ya que es un método que permite estudiar y analizar el comportamiento de las personas en internet, y que permite conocer los problemas y potencialidades que puede tener una organización en el área funcional de marketing. La netnografía es una técnica de investigación que puede analizar y comprender lo que las personas hacen y cómo se relacionan en internet, y también determina los perfiles, características, comportamiento y prácticas habituales de los posibles consumidores 0 clientes cibernautas (Morais, Santos y Gonçalves, 2020). Esta técnica se ha aplicado en análisis de consumidores en línea (Elliott, Shankar, Langer y Beckman 2005), y en esta ocasión se analiza la interacción que están creando los restaurantes con sus clientes por medio de redes sociales.

\section{MARCO TEÓRICO}

Con la masificación de la Internet, ha surgido una nueva forma de comunicación, cuya característica principal es que los individuos no tienen que estar en un mismo lugar, ni de forma sincronizada, interacción que eliminó barreras idiomáticas y también fronteras físicas, facilitando el intercambio de ideas. En ese contexto surgieron las redes sociales, cuyo objetivo es reunir a un grupo de personas que tienen un fin común, logrando contactar a miles de personas con características similares. Lo anterior ha permitido la aparición de espacios virtuales en los que personas que en muchos casos no se conocen se comunican, comparten ideas e interactúan.

El mercadeo en redes sociales no ha sido ajeno a esta dinámica y aunque depende de muchos factores del entorno que lo afectan, ha tenido una evolución vertiginosa, logrando una adaptación al contexto sin precedentes (Castaño, J. J., \& Jurado, S. 2016). Esta evolución fue identificada por el profesor $\mathrm{V}$. Kumar quien a través de una recopilación de hechos identifica con claridad las diferentes etapas del mercadeo, haciendo un análisis de cada una de ellas, 
identificando así sus factores característicos (Kumar, 2015).

En medio de esos cambios, Kumar (2015) explica cómo los nuevos desarrollos de la sociedad impulsan el desarrollo del marketing, haciendo cambiar la estrategia de acuerdo a las condiciones que el mercado le impone a las organizaciones. Uno de esos cambios y quizás el más importante es la influencia que tienen internet, las redes sociales y todo lo relacionado con tecnología, en el comportamiento actual del consumidor.

En redes sociales el mercadeo consiste en un conjunto de diálogos multidireccionales, es participativo y parte (o mucho) de su contenido es generado por los propios consumidores (Galván-Guardiola, 2018). Cartagena (2017) por su parte afirma que las redes sociales ofrecen a las organizaciones una serie de ventajas particulares entre las que se destaca que:

"Las Redes Sociales permiten a las empresas construir marcas a través de diferentes estrategias de comunicación, principalmente mediante la publicación de contenido que ayude a desarrollar una relación personal con cada cliente. Por lo tanto, esta generación y su presencia en Redes Sociales representa una gran oportunidad para el marketing empresarial, en un entorno donde se han fundido la información, la publicidad y el entretenimiento" (Cartagena, 2017).

En este sentido las Redes Sociales Horizontales constituyen el grupo con mayor cantidad de usuarios en el planeta y en el mundo digital. Esto se da particularmente porque los temas que allí se tratan son generales, es decir, no tienen una temática definida para enganchar a sus públicos, por esta razón son los medios de comunicación social más poderosos y pretendidos por las organizaciones comerciales y de servicios. Algunas plataformas de redes sociales que se encuentran en esta clasificación son: Facebook, Twitter, YouTube, WhatsApp, QQ, entre otras.
Si bien hay más canales sociales que nunca, para la empresa resulta fundamental revisar cuáles son los que tienen mayor retorno sobre la inversión (ROI). En el último informe presentado por la empresa Hubspot (2021), se especifica que las redes sociales con mayor ROI en Latinoamérica son Facebook e Instagram.

Por su parte las Redes Sociales Verticales se centran en actividades específicas de los usuarios como, por ejemplo actividades lúdicas, tareas académicas, artes, profesiones, entre otras. También vale la pena destacar que esta clasificación de redes sociales supone un menor número de usuarios cautivos, debido a la especialidad de los temas que se tratan y se comparten en estas. Algunas redes sociales que por sus características son definidas como verticales en el mundo digital son : Linkedln, TripAdvisor, Foursquare, Flickr, Pinterest, GoodReads, entre otras.

Según Comscore (2020) durante la pandemia, en América Latina la red social que ha tenido mayor participación ha sido Instagram, llegando a un $60 \%$, seguido por Facebook. Es de destacar que a pesar de las diferencias culturales, esta red social predomina en las tres regiones que fueron analizadas en el estudio (Asia, Latinoamérica y Europa).

\section{Figura 1.}

Participación de contenido en redes sociales por región
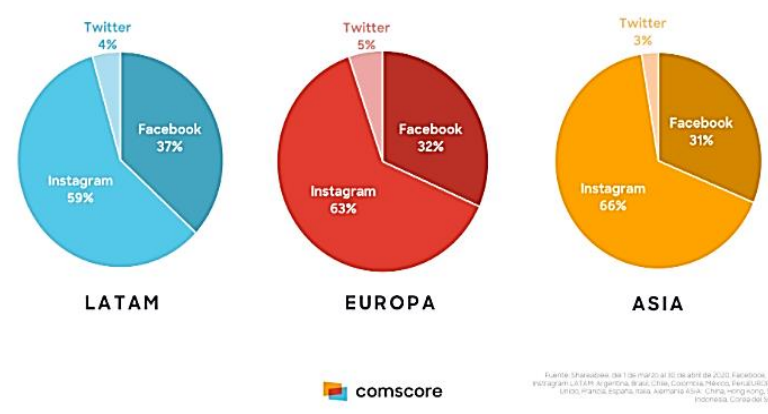

Fuente: Comscore (2020). 
Este comportamiento indica preferencias que durante el confinamiento se vieron realzadas y que confirman que la parte visual, así como la de interacción se convirtió en la más buscada por el público.

\subsection{Diagnóstico de la situación actual del marketing en redes sociales en el sector de los restaurantes en Colombia.}

El año 2020 las búsquedas y menciones referentes a restaurantes en Colombia fueron apaciguadas en una gran magnitud por el COVID-19, una tendencia que se compartió con el Contenido Generado por el Usuario (CGU). En ese contexto lo que han hecho varias marcas y lo que será tendencia en 2021 será la bidireccionalidad de las redes sociales, donde las marcas buscan un diálogo o una conversación con sus clientes.

De esta manera, el marketing conversacional, es decir aquel que logra comunicarse con los clientes generando interacciones por medio de diferentes herramientas como los chatbots, la mensajería social o llamadas telefónicas, busca edificar relaciones más fuertes utilizando contenido personalizado y mucho más directo. Lo anterior no quiere decir que las empresas que no los usan se hayan quedado relegadas, por el contrario, utilizando estrategias de marketing hiperlocales, y focalización lograron sobrevivir a la crisis llegando al consumidor más cercano.

A raíz del confinamiento el sector de los alimentos se vio en la necesidad de buscar opciones para tratar de sobrevivir a la difícil situación, por lo que se vio en las redes sociales una gran oportunidad para comunicarse bidireccionalmente con los usuarios, teniendo en cuenta la penetración que han logrado gracias a la masificación no sólo de dispositivos sino también de conectividad. Es así que la tradición colombiana de pedir alimentos a domicilio se incrementó e hizo que marcas que no lo hacían, incursionaran en esta modalidad para poder seguir compitiendo en el mercado y tratando de sobrevivir a la crisis.
Al respecto, según el diario económico Portafolio (2021), la participación de las ventas a domicilio en el total de las ventas de los restaurantes, pasó del 18\% en el año 2019 a un 45\% en el año 2020, lo que demuestra la importancia de esta alternativa estratégica adoptada por el sector de los restaurantes.

Por otra parte, es importante establecer para entender mejor el análisis realizado en este documento de investigación que la franquicia, basará siempre su fundamento en la comercialización de productos 0 servicios en el marco de criterios de especialización comercial, garantizará la aplicación de modernas técnicas de gestión empresarial en todas las facetas del negocio y, ante todo, constituirá una relación de asociación empresarial que, de acuerdo con principios de autonomía e independencia, faculta a las empresas para rentabilizar su acuerdo mediante la generación de sinergias y economías de escala (Muñoz, 2010). Por lo tanto, es un escenario óptimo para revisar buenas prácticas, en este caso, las que llevan a cabo en sus redes sociales.

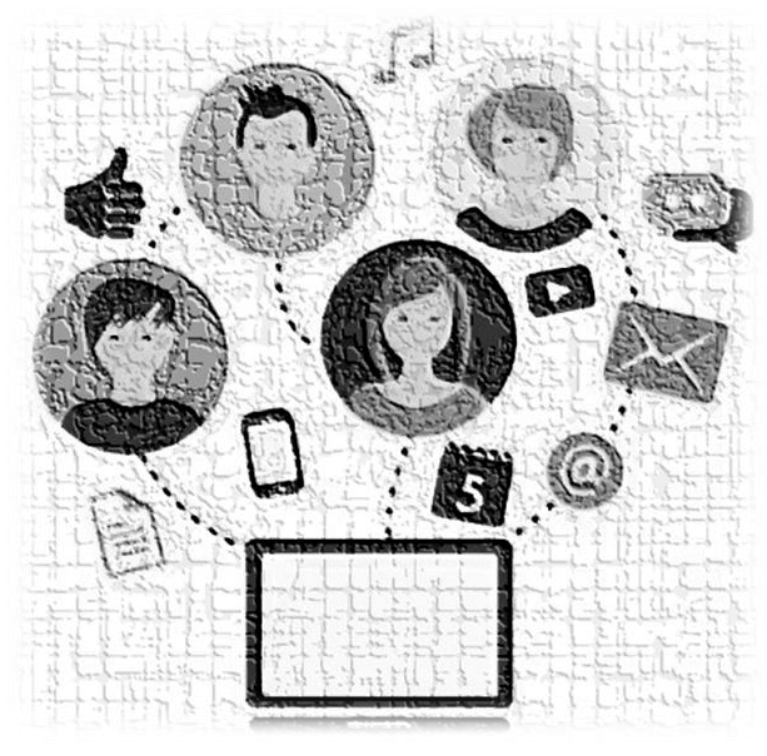




\section{METODOLOGÍA:}

Este documento corresponde a un estudio exploratorio, no experimental, cuantitativo, transversal, inferencial realizado por medio de netnografía. La unidad de análisis o unidad de muestreo son restaurantes formalmente constituidos de pequeño, mediano y gran tamaño según los criterios establecidos en Colombia categorizados en fuentes secundarias como los más representativos (se exceptúan a las microempresas del sector, debido a la informalidad y dificultad en la consecución de los datos en las plataformas). La muestra del estudio está conformada por 146 restaurantes colombianos. Se consideró un nivel de confianza del $95 \%$ con un error de muestra del $5 \%$, teniendo en cuenta que en Colombia existían 90.000 restaurantes antes de la pandemia, cerraron alrededor de 30.000 y sólo aproximadamente el $2 \%$ está catalogado como pequeño, mediano o grande, según el informe presentado por Acodres (2021). La recolección de datos fue mediante el apoyo de semilleros de investigación de diversas universidades quienes consolidaron la información.

Se trabajó una escala de relación para la captura y ajuste a una escala de 10 categorías para cada ítem que permiten conocer el comportamiento de la estrategia en redes sociales. Se tomó en cuenta para el análisis el comportamiento tanto de franquiciados como no franquiciados para hacer los grupos de referencia. Con el fin de procesar los datos se utilizó el programa estadístico software online Wessa y XLSTAT | Software estadístico Excel v 20. Una vez que se probó la bondad de ajuste 0 normalidad de los datos Kolmogorov - Smirnov (K-S), se realizó la prueba correlación de Pearson entre los ítems escogidos. Finalmente se procedió a concluir relacionando los datos cuantitativos y cualitativos encontrados.

\section{RESULTADOS:}

Hallazgos:

\subsection{Análisis de diferencias entre categorías de modelo de negocio (franquiciado 0 no) y correlaciones entre las variables de estudio.}

A partir del nivel de uso de las redes sociales con las que cuentan los restaurantes estudiados, se realizó un análisis de correlación entre las variables que permiten definir la estrategia en redes sociales más adecuada, con el propósito de trazar un camino para que otros restaurantes en estadios más precarios puedan contextualizar las mejores prácticas.

Para alcanzar este objetivo se recolecta por medio de netnografía la información de una muestra de 146 restaurantes colombianos, y se analizan teniendo en cuenta el resultado general, para posteriormente hacer una división entre restaurantes franquiciados o no, esto último con la intención de conocer las fortalezas de la estrategia en redes sociales de empresas franquiciadas que en su mayoría son extranjeras, y pueden contar con mejores prácticas que sean susceptibles de ser adoptadas por el sector. Además, se revisó sistemáticamente de forma cualitativa la información disponible en las redes sociales de los restaurantes en Colombia y se obtuvieron los siguientes resultados: 


\section{Figura 2.}

Contraste de los Perfiles de las Condiciones Necesarias entre restaurantes franquiciados y no franquiciados

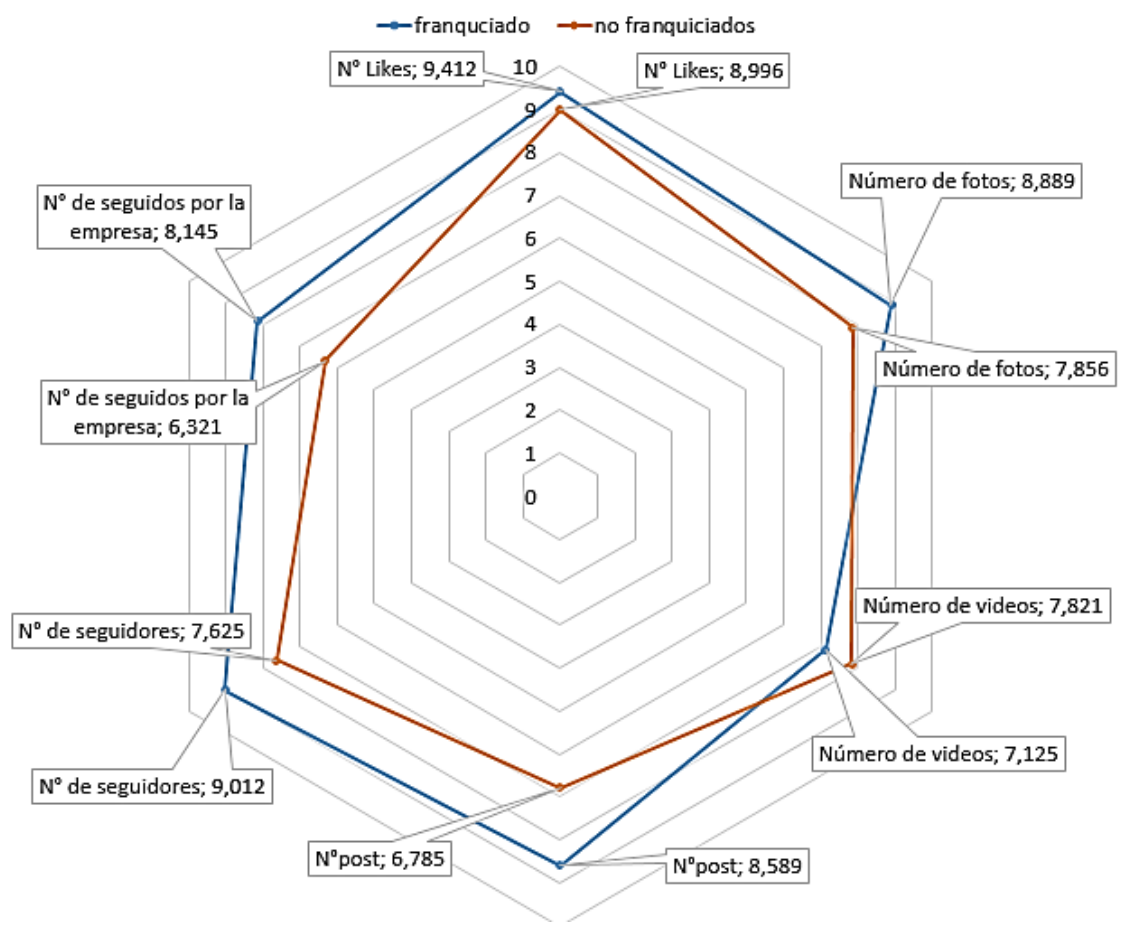

Fuente: Elaboración Propia con base a resultados obtenidos en este estudio.

Nótese que existe una brecha marcada de aproximadamente dos puntos entre las categorías con respecto a dos variables: número de publicaciones y número de seguidores. Esto debido a que las empresas no franquiciadas cuentan en su mayoría con menores recursos (tiempo y dinero), para invertir en la administración de redes sociales, lo cual impacta fuertemente la cantidad de las personas a las cuales les intercambian información de interés por datos personales, fundamentales para la conversión y generación de ingresos para el negocio. Es importante resaltar que las dos variables corresponden a Instagram, una red social más moderna que Facebook, la cual reúne a una nueva generación familiarizada con los entornos digitales.
Por otro lado, se observa una pequeña diferencia $(0,42)$ entre las dos categorías (franquiciados y no franquiciados) en la variable número de likes o me gusta. El Número de likes representa en Facebook el número de leads generados por el restaurante en su página, lo cual nos muestra que esta plataforma está siendo administrada de una manera similar en ambas categorías y por lo tanto, sería más fácil realizar un proceso de Lead Nurturing, es decir, entender en qué fase del ciclo de compra se encuentra el cliente para ofrecerle contenidos que le motiven a mantener el interés en el restaurante $y$, finalmente, a tomar una decisión de compra final. 
Los resultados obtenidos de forma cuantitativa y cualitativa, reflejan que para el sector hay oportunidad de mejora, sin embargo, habría que establecer correctamente la estrategia 0 criterio seguir contextualizado a cada caso para mejorar la gestión de las redes sociales, las cuales pueden abordarse de tres formas:

se debe incluir contenido en Reels, IGTV o Facebook live.

2) Cerrar las brechas en los ítems que muestran mayor diferencia de opinión de los grupos (número de post y número de seguidores). Esto solo es posible si se invierte tiempo y recursos en dos elementos fundamentales: creación de contenido y mayor engagement, representado por menciones, comentarios y aumento de las veces en la que la publicación es compartida.

3) Considerar las dependencias entre las variables, esto es las correlaciones existentes, y de esta manera generar una aproximación sobre el efecto que tienen la modificación de una de las variables sobre el

\begin{tabular}{|c|c|c|c|c|c|c|}
\hline & $\mathrm{N}^{\circ}$ Likes & $\begin{array}{l}\text { Número de } \\
\text { fotos }\end{array}$ & $\begin{array}{l}\text { Número de } \\
\text { videos }\end{array}$ & $\mathrm{N}^{\circ}$ Post & $\mathrm{N}^{\circ}$ de Seguidores & $\begin{array}{c}\mathrm{N}^{\circ} \text { de seguidos por la } \\
\text { empresa }\end{array}$ \\
\hline $\mathrm{N}^{\circ}$ Likes & 1,0000 & $0,6250^{*}$ & 0,5891 & $0,6012^{\star *}$ & $0,6182^{*}$ & 0,5012 \\
\hline Número de fotos & & 1,0000 & $0,5799^{*}$ & 0,4123 & $0,7584^{\star *}$ & 0,5512 \\
\hline Número de videos & & & 1,0000 & 0,5593 & 0,4512 & $0,6731^{*}$ \\
\hline $\mathrm{N}^{\circ}$ Post & & & & 1,0000 & 0,3526 & 0,4125 \\
\hline $\mathrm{N}^{\circ}$ de seguidores & & & & & 1,0000 & 0,1256 \\
\hline $\begin{array}{l}\mathrm{N}^{\circ} \text { de seguidos por la } \\
\text { empresa }\end{array}$ & & & & & & 1,0000 \\
\hline
\end{tabular}

Fuente: Elaboración Propia con base a resultados obtenidos en este estudio. *; **, es una correlación es significante al nivel 0,05 Y 0,01 respectivamente.

1) Mejorar en las dimensiones que se identificaron como más débiles para ambas categorías seguidos por la empresa y números de videos. Los seguidos por la empresa son un recurso poco conocido pero muy utilizado para procesos de posicionamiento de marca, debido a que al enviar una solicitud de seguimiento por medio de Instagram a usuarios que estén interesados en productos de mi competencia, es muy probable que revisen la página del restaurante y lo sigan, o que al menos tengan que estar expuestos a ver mi marca. Asimismo, los videos son un recurso ampliamente utilizado en la actualidad y un formato con gran aceptación por parte de los usuarios, motivo por el cual
La tabla 1 se obtuvo utilizando el programa XLSTAT, en ella se pueden observar los 36 índices de correlación posibles entre las 6 variables de estudios (ver tabla 2):

Solo 8 casos detectados de correlación encontrados, entre los 6 ítems son significativos (Sig. Mayor a 0.05). Tenemos 5 índices de correlación mayores a 0.6 denominados correlación fuerte en los siguientes casos: (Número de likes y fotos) ;( Número de likes y Número de post); (Número de likes y Número de seguidores) ;(Número de fotos y Número de seguidores) y (Número de videos y Número de 
seguido). Existen además índices de correlación mayores a 0.55 pero menores a 0.6 denominados moderados y son los siguientes (Número de likes y Número de videos); (Número de fotos y Número de videos) (Número de fotos y Número de seguidos) y (Número de videos y Número de Post). La tabla 2 presenta el conteo y clasificación realizada de los demás pares.

Tabla 2.

Conteo de las Correlaciones de Pearson encontradas

\begin{tabular}{lccccc}
\hline İtems & Fuertes & Moderada & Moderada - & Débiles (< & Interrelaciones \\
& $(>0.6)$ & $(>0.55)$ & débil $(>0.5)$ & $0.5)$ & significativas \\
\hline $\mathrm{N}^{\circ}$ Likes & 3 & 1 & 1 & 0 & 3 \\
Número de fotos & 2 & 2 & 0 & 1 & 2 \\
Número de videos & 1 & 3 & 0 & 1 & 1 \\
$\mathrm{~N}^{\circ}$ Post & 1 & 1 & 0 & 3 & 1 \\
$\mathrm{~N}^{\circ}$ de Seguidores & 2 & 0 & 0 & 3 & 2 \\
$\mathrm{~N}^{\circ}$ de Seguidos por la empresa & 1 & 1 & 1 & 2 & 1 \\
\hline
\end{tabular}

Fuente: Elaboración Propia con base a resultados obtenidos en este estudio.

Con esta información se procedió a construir un diagrama (figura 3) que ayuda a comprender con mayor claridad la información obtenida. El diagrama presenta de manera sencilla una representación de una de las 6 variables, el tamaño de cada círculo está representado por el valor que se muestra en la figura 1 , de tal manera que círculos más grandes (Número de likes,) indican que la dimensión tiene un valor mayor y círculos pequeños (Número de fotos) indican que el valor fue más bajo. La correlación está dada en este caso por el patrón de color.

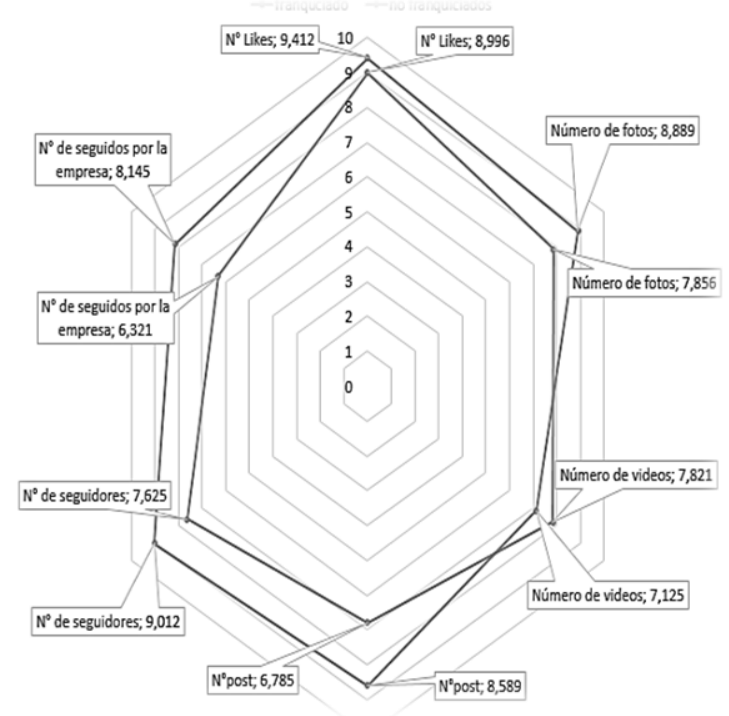




\section{Figura 3}

Diagrama de Correlación observada entre las 6

variables del estudio

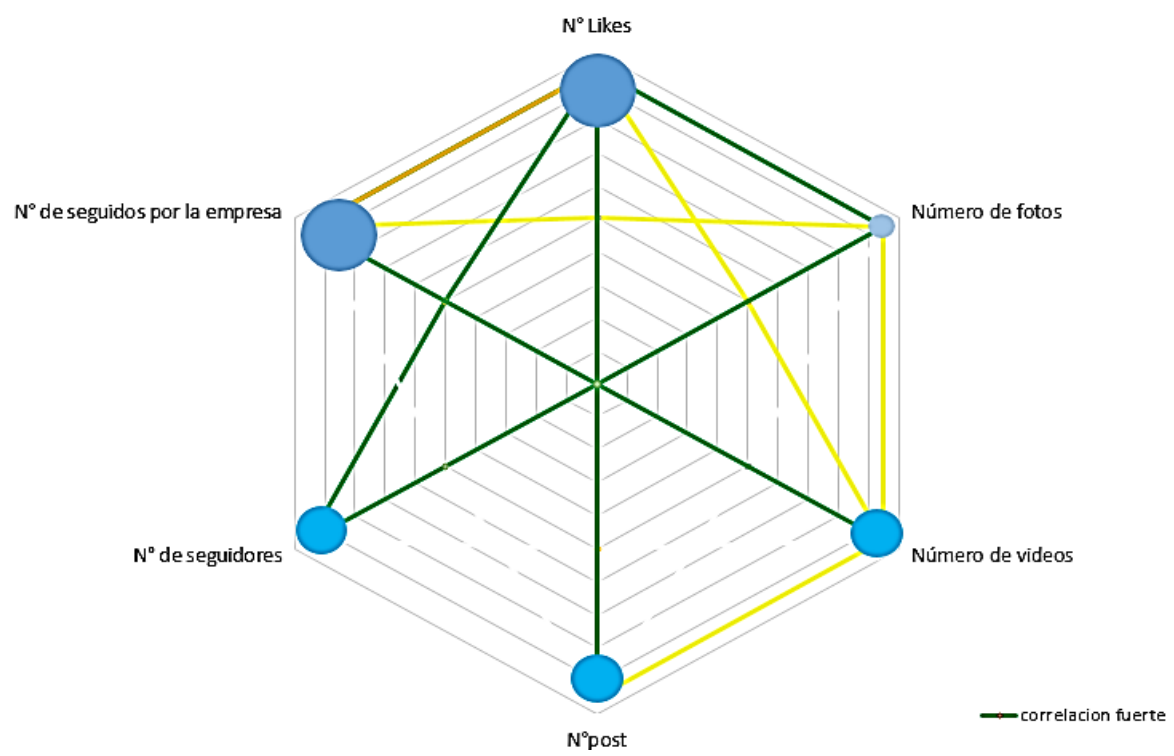

Fuente: Elaboración Propia con base a resultados obtenidos en este estudio.

\subsection{Análisis de las buenas prácticas aplicadas por el sector de los restaurantes en la gestión de las redes sociales Facebook e Instagram.}

\subsubsection{Facebook}

interrelaciones genera es la Número de likes (9.61), y tiene tres correlaciones fuertes (número de post, Número de seguidores y número de fotos) una correlación moderadas (número de vídeos) y una débil con (número de seguidos por la empresa). Otra relación que confirma este estudio es que el número de seguidores (9.230), tiene dos correlaciones fuertes, lo que indica que el mejoramiento de esta variable se podría impactar positivamente en el número de likes y la categoría de fotos, como se ha podido observar en la evidencia empírica. 
para tener una comunicación más fluida entre cliente y restaurante. También debe contar con el correo empresarial y un vínculo con la página web de la empresa.

Otro punto clave es la publicación de fotos con los platos disponibles, por lo tanto, se decidió analizar la evolución de las publicaciones desde dos puntos de vista; en primer lugar, cuando el restaurante comienza a postear fotos de sus platos y su estructura física, la calidad y la proporción de la imagen generalmente no son buenas, las fotos carecen de luz y la edición es casera, adicionalmente no eran constantes con la publicación de fotos en ésta red social. En comparación de ello, se evidencia que, desde hace tres años, la importancia que tiene la publicidad visual para este tipo de empresas tuvo una mejora notoria, los posters tienen una mejor edición, respetando la paleta cromática de color en cada uno de ellos, la calidad y proporción de las imágenes mejoró sustancialmente y en promedio publican dos fotos por día.

Otro punto a tratar hace referencia a la cantidad de "Me gusta" que tiene cada publicación; cuando algunos restaurantes comenzaron a publicar imágenes, no tenían "Me gusta" ni "Comentarios" demostrando que no había aceptación por parte de los seguidores. En la actualidad, las publicaciones tienen un promedio de 50 a 150 "Me gusta" y no pasan de 20 comentarios. De acuerdo a lo anterior se puede afirmar que aún no se presenta una buena aceptación por parte de los seguidores de las páginas.

Los restaurantes han empezado a publicar en formato videos, desde hace aproximadamente cinco años; en los que informan qué actividades familiares, de amigos o pareja, tiene el restaurante. Los videos de la mayoría de restaurantes poseen una buena edición y una buena calidad, sin embargo, es importante resaltar que no son publicaciones constantes y que al igual que con las fotos, la aceptación de los seguidores no es la mejor. En este aspecto, se sugiere ser más interactivo, mostrar aspectos de la compañía mediante videos elaborados, por ejemplo ¿Cómo se vive la experiencia de estar en el restaurante?, teniendo relación con los clientes y demás servicios que ofrece el restaurante.

Por ser empresarial, la página de Facebook tiene un ítem de "opinión", en donde los seguidores pueden comentar acerca de su experiencia; en casos específicos, por ejemplo, los seguidores han compartido en promedio 602 opiniones. Analizando dichos comentarios, el $68 \%$ hacen referencia a que la experiencia no fue buena, mala atención al cliente y mala calidad de los productos, información valiosa para encontrar aspectos a mejorar. Hay muchas personas que afirman haberse dirigido a las oficinas 0 administraciones de los restaurantes demostrando su desacuerdo, sin recibir respuesta alguna. Con base en ello, se cree que es pertinente una retroalimentación de los empleados y el gerente de la empresa, con el fin de solucionar dichas inconformidades que posee el cliente y no aumentar una mala reputación por medio de las redes sociales, ya que provienen de perfiles reales y poseen credibilidad, esto puede generar que personas que posiblemente no han ido al restaurante se hagan una imagen negativa del establecimiento y no estén dispuestos a visitarlo.

Para concluir, últimamente se evidencia que la actividad que tienen en esta red social es constante por parte del restaurante y de los usuarios, sin embargo, se sugiere que con estrategias de publicidad y de interacción con los seguidores, tendrán la capacidad de crecer más. Es importante tener en cuenta que las opiniones que dejan las personas en la página de Facebook, deben ser respondidas en su totalidad, esto permite una confianza entre el seguidor y la empresa, ya que se identificó que sólo responden las opiniones buenas que dejan de los restaurantes.

\subsubsection{Instagram}

Del mismo modo se encuentra que los restaurantes poseen Instagram como una de sus redes sociales. Es evidente que también posee una cuenta (profesional) en el apartado de empresas en la plataforma llamada 


\section{ANÁLISIS DE ESTRATEGIA EN REDES SOCIALES PARA EL SECTOR DE LOS RESTAURANTES COLOMBIANOS EN TIEMPOS DE CRISIS POR LA PANDEMIA COVID-19 \\ Yenny Katherine Parra Acosta - Carlos Alberto Almanza - Diego Felipe Astudillo Valverde}

Instagram con la arroba correspondiente, la cual viene registrando actividad en su mayoría desde el año 2015, contando hoy en día los restaurantes con miles de seguidores y más de tres mil publicaciones en promedio, donde se comparte información relativa a los restaurantes. También se identificaron botones para poder interactuar con la información de la empresa los cuales parecen muy oportunos a la hora de querer informarse más sobre la misma.

Instagram desde su apertura en el año 2010 ha venido creciendo y expandiéndose a medida que el tiempo pasa de una manera extraordinaria, siendo hoy una de las redes sociales más grandes e importantes contando con alrededor de mil millones de usuarios activos alrededor del mundo, y que para muchos se postula como la red social favorita en Latinoamérica, donde pasan una cantidad de tiempo considerable cada día, viendo fotos y videos de acuerdo a su interés. De esta manera, Instagram se ha convertido en una opción muy atractiva para las empresas en cuanto a encontrar clientes potenciales para sus productos 0 servicios se refiere, logrando esto por medio de publicidad sectorizada hacia personas con intereses en común relacionados con el oficio de las empresas.

De acuerdo a lo anterior, el perfil empresarial en Instagram de los restaurantes cuenta en promedio con más de tres mil publicaciones, las cuales están alineadas con el objetivo que busca la empresa en esta red social que es dar a conocer sus productos y servicios. Se visualiza que las publicaciones, distribuidas entre imágenes y videos, son bastante atractivas y llevan una información continua valorando que en muchas ocasiones las publicaciones se tratan de marketing de contenido, elemento que ha mostrado su efectividad en los últimos años.

Las publicaciones acerca de eventos que realizan los restaurantes con temáticas específicas, como por ejemplo, días especiales relacionados con comida especial, celebraciones de fechas como el día de amor y amistad, el día de la madre o también se encuentran eventos como los partidos de fútbol, invitando al cliente a verlos en los restaurantes, y asimismo se encuentran publicaciones de especialidades en platos de comida, promociones como descuentos por pagar con tarjeta de crédito, y promociones permanentes.

Otro punto importante a resaltar del perfil de los restaurantes en Instagram son los diferentes botones que se encuentra en la parte superior o encabezado de la página, donde el potencial cliente obtendrá la oportunidad de interactuar en la página con el fin de obtener información, hacer reservas y también se encuentran apartados para enviar mensajes a la empresa y llamar directamente a la línea de atención al cliente. También se encuentra el segmento donde se ve la ubicación del negocio por medio de una aplicación de mapas. En este mismo segmento se ubican publicaciones de clientes que suben contenidos en la empresa donde se puede conocer un poco más de información de la relación entre el cliente y los servicios o productos obtenidos.

En conclusión el perfil empresarial en Instagram que mantienen los restaurantes está muy completo, seguramente debido a una transformación digital acelerada por el COVID-19, pues cuenta con la información pertinente de los movimientos de la empresa, con los accesos para poder contactar a la empresa, poder hacer reservaciones, saber la ubicación, entre otros anteriormente mencionados, y desde luego hay que resaltar la calidad en dichas publicaciones donde denotan un trabajo de producción de la misma antes de ser subida a la cuenta, sin embargo, se demuestra que sus seguidores mantienen una interacción mínima con las publicaciones y/o un interés bajo por la información ubicada en la página.

\section{DISCUSIÓN Y CONCLUSIONES:}

Después de realizar una revisión netnográfica de las dos redes sociales con mayor retorno sobre la inversión en Latinoamérica, para el sector de los restaurantes en Colombia se puede concluir que: 
Las empresas no franquiciadas poseen en su mayoría menos recursos, principalmente capital humano capacitado y dinero, para invertir en la administración de redes sociales, lo cual impacta fuertemente la cantidad de las personas a las cuales les intercambian información de interés por datos personales, fundamentales para aumentar la conversión, la generación de ingresos para el negocio y obtener campañas promovidas por los clientes. Es importante resaltar que las dos variables corresponden a Instagram, una red social con un público más familiarizado con los entornos digitales que Facebook.

La plataforma Facebook está siendo administrada de una manera similar en ambas categorías (franquiciados y no franquiciados), y por lo tanto, sería ideal realizar un proceso de Lead Nurturing en el cual se identifica en qué fase del ciclo de compra se encuentra el cliente para ofrecerle contenidos que le motiven a mantener el interés en el restaurante, y finalmente, a tomar una decisión de compra que posteriormente volverse un promotor de la marca.

Los seguidos por la empresa son un recurso poco conocido pero muy utilizado para procesos de posicionamiento de marca, debido a que al enviar una solicitud de seguimiento por medio de Instagram a usuarios que estén interesados en productos de mi competencia, es muy probable que revisen la página del restaurante y lo sigan, o que al menos tengan que estar expuestos a ver mi marca. Asimismo, los videos son un recurso ampliamente utilizado en la actualidad y un formato con gran aceptación por parte de los usuarios, motivo por el cual se debe incluir contenido en Reels, IGTV o Facebook live. Además, se debe invertir tiempo y recursos en dos elementos fundamentales: creación de contenido y creación de mayor engagement, representado por menciones, comentarios y aumento de las veces en la que la publicación es compartida. Por último, cuantitativamente se confirmó la correlación entre la cantidad de publicaciones, establecida en Facebook por el número de fotos principalmente y el número de leads o como se nombran en Facebook "likes" o me gusta, situación que sugiere la importancia de la regularidad en las publicaciones.

De forma cualitativa, también se pudo establecer que la actividad constante y respuesta oportuna a los comentarios positivos que los clientes hacen a las empresas del sector en Facebook e Instagram es un factor importante de fidelización y monetización de las campañas publicitarias que se llevan a cabo. Sin embargo, es importante tener en cuenta que las opiniones que dejan las personas en la página de Facebook, deben ser respondidas en su totalidad, eso permite una confianza entre el seguidor y la empresa, ya que se identificó que normalmente sólo responden las opiniones positivas y eliminan las negativas.

Una estrategia que podrían implementar los restaurantes puede ser la interacción con los clientes por medio de Facebook, por ejemplo, si la publicación específica llega a un determinado número de "Me gusta" se realizará una actividad con música en vivo 0 se llevarán a cabo promociones en las bebidas. De esta forma será un incentivo a que los seguidores interactúen con la página creando una comunidad más sólida y aumentando la popularidad de la empresa.

Otro punto a mejorar es la interacción entre el usuario y la pagina ya que a pesar de que poseen un gran número de seguidores, su mayoría de publicaciones contienen alrededor de 30 "Me gusta" por publicación, esto demuestra que, sin importar esta cantidad de seguidores, los mismos no interactúan con las páginas, y no demuestran un interés en conocer la información de las empresas. Un problema que aparece además es que los intereses de los seguidores no coinciden con los temas que comparten las páginas. Es recomendable hacer campañas pagas donde aquellas se compartan a potenciales clientes con gustos por la gastronomía específica, este pago por publicidad que se realiza en la plataforma se puede realizar de manera progresiva, y entre más seguido se efectúe, mayor participación de mercado se obtendrá. 
El perfil empresarial que mantienen los restaurantes pequeños, medianos y grandes en Facebook e Instagram, en su mayoría están muy completos, con la información pertinente de los movimientos de la empresa, con los accesos para poder contactar a la empresa, hacer reservaciones, saber la ubicación, entre otros accesos. Desde luego hay que resaltar la calidad en dichas publicaciones donde denotan un trabajo de producción de la misma antes de ser subida a la cuenta, todo esto debido en gran parte a la transformación digital acelerada por motivo del COVID19. Sin embargo, se demuestra que sus seguidores mantienen una interacción mínima con las publicaciones y/o un interés bajo por la información ubicada en la página.

Asimismo, los diferentes botones que se encuentra en la parte superior o encabezado de la página, donde el potencial cliente obtendrá la oportunidad de interactuar en la página con el fin de obtener información, como por ejemplo se encuentra un enlace al sitio web donde puede realizar una reservación, también se encuentra apartados para enviar mensajes a la empresa y para llamar directamente a la línea de atención al cliente y, desde luego, también se encuentra el segmento donde se ve la ubicación del negocio por medio de una aplicación de mapas, en este mismo segmento tiene publicaciones de clientes que suben fotos o videos en la empresa donde se puede conocer un poco más de información de la relación entre el cliente y los servicios o productos obtenidos.

Los resultados obtenidos en este estudio, reflejan que en el sector hay oportunidad de mejora, sin embargo, habría que establecer correctamente la estrategia 0 criterio contextualizado a cada caso para mejorar la gestión de las redes sociales.

\section{REFERENCIAS:}

Barzola, L., Jara, J., \& Aviles, P. (2019). Importancia del Marketing Digital en el Comercio Electrónico. E-IDEA Journal of Business Sciences, 1(3), 24-33.

Castaño, J. J., \& Jurado, S. (2016). Marketing digital (Comercio electrónico). Editex.

Cartagena, D. J. (2017). Millennials y redes sociales: estrategias para una. Miguel Hernández Communication Journal, 355.

Comscore (2020). Análisis Global: El Efecto de la Pandemia del Coronavirus en Social Media. Recuperado de: https://www.comscore.com/lat/Prensa-yEventos/Blog/Analisis-Global-El-Efecto-dela-Pandemia-del-Coronavirus-en-SocialMedia

Conti, E., \& Lexhagen, M. (2020). Instagramming nature-based tourism experiences: a netnographic study of online photography and value creation. Tourism Management Perspectives, 34, 100650.

David, F. R. (2003). Conceptos de administración estratégica. Pearson Educación.

Elliott, R., Shankar, A., Langer, R., \& Beckman, S. C. (2005). Sensitive research topics: netnography revisited. Qualitative market research: An international journal.

Espinoza López, J. A., \& Ramírez Flores, D. M. (2019). Diseño de estrategias de marketing digital para el restaurante Leos de Mariscos del Cantón Naranjal (Bachelor's thesis, Universidad de Guayaquil Facultad de Ciencias Administrativas).

Galván-Guardiola, Y. Y. (2018). Redes sociales y tendencias de marketing digital en los negocios. Latindex, 702. 
Hubspot (2021). Informe estado actual de marketing digital. recuperado de: https://www.hubspot.es/estado-del-marketing

Kotler, F., \& Armstrong, G. (2015). Fundamentals of marketing. Moscow: Progress.

Kozinets, R. V. (2007). Netnography. The Blackwell Encyclopedia of Sociology, 1-2.

Linares Cazola, J. G., \& Pozzo Rezcala, S. K. (2018). Las redes sociales como herramienta del marketing relacional y la fidelización de clientes.

Matosas-López, L. (2021). The Management of Digital Marketing Strategies in Social Network Services: A Comparison between American and European Organizations. Journal of Open Innovation: Technology, Market, and Complexity, 7(1), 65.

Muñoz (2010). La Franquicia, una estrategia de crecimiento empresarial. recuperado de: https://www.eafit.edu.co/revistas/revistamba/ Documents/franquicia-estrategiacrecimiento-empresarial.pdf

Kumar, V. (2015). Evolution of Marketing as a Discipline: What Has Happened and What to Look Out For. Journal of Marketing, 79(1), 19. Retrieved July 23, 2020, from www.jstor.org/stable/43784378

Michael, F. F. M. B. U., Work, E. P. S., \& Porter, M. Michael E. Porters Five Forces Model E-book From Wikipedia. Innovation, 15(4.2), 3.

Montenegro Delgado, L. A. (2018). Estrategias de marketing relacional para el posicionamiento de la marca del restaurante-Cevicheria el Burrito-Lambayeque, 2016.

Morais, G. M., Santos, V. F., \& Gonçalves, C. A. (2020). Netnography: Origins, Foundations, Evolution and Axiological and Methodological Developments and Trends. The Qualitative Report, 25(2), 441-455.

Patro, C. S. (2021). Digital Tourism: Influence of EMarketing Technology. In Impact of New Media in Tourism (pp. 234-254). IGI Global.
Portafolio (2021). En 2020 el 45\% de servicios de comida fueron domicilios. Recuperado de: https://www.portafolio.co/economia/aplicacio nes-rappi-o-ifood-en-2020-el-45-por-cientode-servicios-de-comida-fueron-domicilios548413

Quispe, V., \& Linda, R. (2019). Marketing relacional enfocado a la fidelización de los clientes del restaurante mar criollo, Lima 2019.

Semana (2021). Cuántos bares y restaurantes han cerrado por el coronavirus. Recuperado de: https://www.semana.com/empresas/articulo/ cuantos-bares-y-restaurantes-han-cerradopor-el-coronavirus/299647/ 